Investigación

\title{
ANÁLISIS DE LAS HABILIDADES MATEMÁTICAS PARA EL CÁLCULO DE DOSIS DE MEDICAMENTOS EN ESTUDIANTES DE ENFERMERÍA
}

\author{
ANALYSIS OF MATHEMATICAL SKILLS FOR THE CALCULATION OF \\ MEDICINES DOSES IN NURSING STUDENTS
}

\begin{abstract}
Alvaro Luis Fajardo Zapata
Médico cirujano

Doctor en Ciencias de la educación, Magíster en Toxicología, Magíster en Salud Pública. Profesor asociado, Departamento de Ingeniería y Ciencias Básicas, Fundación Universitaria del Área Andina. Bogotá, Colombia

https://orcid.org/0000-0001-6067-3053

afajardo@areandina.edu.co
\end{abstract}

\section{Nidia Patricia Caicedo Murte}

Enfermera

Fundación Universitaria del Área Andina. Bogotá, Colombia

https://orcid.org/0000-0003-0303-8306

pattycaicedo02@gmail.com

$\overline{\text { Artículo recibido el } 28 \text { de octubre de 2021. Aceptado en versión corregida el } 15 \text { de diciembre }}$ de 2021 .

\section{RESUMEN}

INTRODUCCIÓN: Los estudiantes de enfermería deben desarrollar habilidades matemáticas para una vez que sean profesionales de esta disciplina no tengan obstáculos con la dosificación de medicamentos, que es una de las funciones que deben desarrollar en el ámbito clínico, y cuya equivocación podría poner en riesgo la seguridad y vida del paciente. OBJETIVO: Analizar de qué manera las habilidades para las matemáticas básicas afecta la realización del cálculo de las dosis de medicamentos por parte de los estudiantes de enfermería en una institución universitaria. MATERIALES Y MÉTODOS: investigación descriptiva transversal realizada a través del instrumento "Habilidad matemática para el cálculo de las dosis de medicamentos" compuesto por 13 preguntas abiertas y aplicado a 256 estudiantes de los ocho semestres que componen un programa de enfermería. RESULTADOS: El estudio evidenció serias deficiencias en la resolución de situaciones que involucran distintas habilidades matemáticas básicas que debe poseer un estudiante de enfermería. Solo el 30,7\% de los estudiantes pudo resolver las situaciones clínicas en las cuales tenía que realizar el cálculo de las dosis de medicamentos; también en el manejo de los porcentajes se encontró dificultades, ya que apenas el 42\% logró resolver la situación planteada. La interpretación de conceptos matemáticos básicos mediante la utilización de 
Análisis de las habilidades matemáticas para el cálculo de dosis ... gráficos fue interpretada adecuadamente por el 50,2\%. CONCLUSIÓN: Los hallazgos de la presente investigación, mostraron que deben buscar estrategias de aprendizaje que mejoren las habilidades de los estudiantes de enfermería para la dosificación de medicamentos.

Palabras clave: administración de fármacos, estudiante de enfermería, habilidades matemáticas, cálculo de fármacos, equivocación en la medicación, seguridad del paciente.

\begin{abstract}
INTRODUCTION: Nursing students must develop mathematical skills so that once they are professionals in this discipline, they do not have obstacles with the dosage of medications, which is one of the functions that they must develop in the clinical field, and whose error could put at risk the safety and life of the patient. OBJECTIVE: To analyze how the skills for basic mathematics affect the calculation of medicine doses by nursing students in a university institution. MATERIALS AND METHODS: descriptive cross-sectional research, carried out through the instrument "Mathematical ability to calculate drug doses" made up of 13 open-ended questions and applied to 256 students from the eight semesters that make up a nursing program. RESULTS: The study showed serious deficiencies in the resolution of situations that involve different basic mathematical skills that a nursing student must possess. Only $30.7 \%$ of the students were able to resolve the clinical situations in which they had to perform the calculation of the drug doses; Difficulties were also found in managing the percentages, since only $42 \%$ managed to resolve the situation. The interpretation of basic mathematical concepts using graphics was adequately interpreted by $50.2 \%$.
\end{abstract} CONCLUSION: The findings of the present investigation showed that they should seek learning strategies that improve the skills of nursing students for the dosage of medications.

Keywords: Drug administration, nursing student, math skills, drug calculation, medication errors, patient safety.

\title{
http://dx.doi.org/10.7764/Horiz_Enferm.32.3.283-296
}

\section{INTRODUCCIÓN}

La seguridad del paciente es un principio fundamental en la atención en salud y es responsabilidad de todas aquellas personas que se involucran directa o indirectamente con su atención. Por tal razón, se deben evitar las equivocaciones que puedan poner en riesgo la integridad y aún la vida del paciente. De acuerdo con la organización Mundial de la Salud OMS, uno de cada trescientos pacientes sufre daños ocasionados por la atención médica, di- chos daños ocupan el $14^{\circ}$ lugar entre las causas de morbi-mortalidad mundial. Afirma esta Institución que, de los aproximados 421 millones de las personas que se hospitalizan cada año en el mundo, 42,7 millones sufren eventos adversos relacionados con la estancia hospitalaria. Además, se calcula, que, en los países de ingresos altos, uno de cada diez pacientes sufre algún tipo de daño durante el tiempo que permanece hospitalizado, daño que se 
podría prevenir hasta en un $50 \%$. Sumado a lo anterior, se calcula que el $15 \%$ de la actividad y del gasto total de los hospitales de los países que conforman la Organización para la Cooperación y el Desarrollo Económico (OCDE), son consecuencia directa de los eventos adversos ${ }^{(1)}$.

Las equivocaciones en la atención de los pacientes a nivel clínico han sido abordadas por varios estudios que han concluido que están relacionados con múltiples factores dentro de los que se mencionan: acumulación de actividades, personal insuficiente, cualificación insuficiente del personal de salud, interrupciones frecuentes en la preparación de los medicamentos, presencia de ruidos, falta de iluminación, aspectos propios de la infraestructura y dotación hospitalaria. También se han evidenciado equivocaciones por parte del personal de enfermería en: administración de medicamentos diferentes a los prescritos; equivocaciones en el cálculo de dosis; administración de medicamentos no autorizados y la alteración de los horarios de administración de estos ${ }^{(2,3,4,5,7)}$.

En esa misma línea, una de las equivocaciones más señaladas es la dosificación y administración de medicamentos. Se define equivocación o error en la medicación, como cualquier suceso evitable que se da por la utilización inadecuada de medicamentos vinculados con la práctica profesional, y que son producto de la actividad equivocada o sin fundamento que puede influir de manera negativa en la salud de los pacientes. Las fallas en la disposición hasta la ejecución de una orden médica pueden transitar por diferentes fases que van desde la vía de administración, el tiempo de duración del tratamiento, la frecuencia de dosificación, un cálculo inadecuado de la dosificación que no toma en cuenta la edad y el peso del paciente, las comorbilidades, o la interacción del medicamento prescrito con otro que concomitantemente debe recibir el paciente para otro tipo de patología ${ }^{(8)}$.

Los estudiantes de enfermería en su proceso formativo deben desarrollar habilidades matemáticas para que una vez sean profesionales de esta disciplina, no tengan obstáculos con el cálculo de las dosis de medicamentos, que es una de las funciones que deben desarrollar en el ámbito clínico, y cuya equivocación podría poner en riesgo la seguridad y vida del paciente. Una educación bien fundamentada es la clave para garantizar que estos estudiantes se conviertan en profesionales seguros e idóneos. Una revisión de la literatura sobre diferentes enfoques para enseñar y evaluar el cálculo de medicamentos con estudiantes de enfermería reveló tres factores principales que influyeron en la capacidad para calcular medicamentos con precisión, estos factores incluyeron la capacidad matemática, particularmente al multiplicar con decimales, comprender fórmulas de medicamentos y conceptualizar la dosis de medicamentos $^{(9)}$.

Un estudio llevado a cabo por Özyazıcıŏglu ${ }^{(10)}$, en Turquía cuyo objetivo fue evaluar el conocimiento de los estudiantes de enfermería sobre los cálculos de dosis pediátricas, encontró que los estudiantes contestaron correctamente el $87,1 \%$ de las preguntas, mientras que el 9,5\% respondió incorrectamente y el 3,4\% dejó el test en blanco. Se encontró que los estudiantes fallaron en el cálculo cuando había fracciones decimales. Este resultado 
Análisis de las habilidades matemáticas para el cálculo de dosis ...

mostró que, los cálculos de dosis que se basan valores decimales pueden generar muchas más equivocaciones. Agregado a lo anterior, Gorgich ${ }^{(5)}$, en la república de Irán, en una investigación cuyo propósito fue indagar las causas de las equivocaciones en la medicación y las estrategias para prevenirlos en enfermeras y estudiantes, encontró que las causas más comunes en enfermeras fueron el cansancio debido a la sobrecarga laboral (97,8\%); y en estudiantes, el cálculo de la dosis de medicamentos (77,4\%). De acuerdo con la opinión de los participantes en la investigación, la mejor forma de prevenir las equivocaciones es reducir la presión laboral y aumentar el personal proporcionalmente al número y condición de los pacientes, así como también, crear una unidad de cálculo de medicamentos.

Un estudio sobre el cálculo numérico de dosis de medicamentos llevado a cabo en el Reino Unido por McMullan ${ }^{(11)}$, mostró que, el $92 \%$ de las estudiantes y el $89 \%$ de las enfermeras no superaron la prueba de cálculo de medicamentos. Este estudio no encontró diferencias significativas entre los estudiantes y enfermeras graduadas en su capacidad para el cálculo de las dosis de medicamentos. El estudio concluye que, para evitar las posibles equivocaciones en la práctica clínica, las enfermeras graduadas deben continuar practicando los cálculos de dosis de medicamentos, que se debe reservar tiempo en los planes de estudio para que los estudiantes de enfermería aprendan cómo realizar cálculos numéricos básicos y cálculos de medicamentos. Este aprendizaje debe ser reforzado a través de la práctica regular y la evaluación.
Asimismo, Yapucu ${ }^{(12)}$, en Turquía, en una investigación que buscaba conocer las habilidades para el cálculo de dosis de medicamentos en estudiantes de dos escuelas de enfermería, encontró que estos tenían habilidades bajas. También Grugnetti ${ }^{(12)}$, en Italia, en un estudio para verificar si el uso de la calculadora en una prueba de habilidad matemática escrita reducía las equivocaciones en el cálculo de dosis de medicamentos en estudiantes de enfermería, encontró serias deficiencias en los cálculos matemáticos a pesar del uso de la calculadora. Igualmente, Bagnasco ${ }^{(13)}$, en Italia, exploró las dificultades de los estudiantes de enfermería en matemáticas e identificó intervenciones educativas adecuadas para cerrar las brechas de conocimiento; éste investigador identificó que los estudiantes universitarios de enfermería tenían dificultades principalmente con los principios básicos de matemáticas, además concluyó que se necesitan intervenciones de aprendizaje específico para mejorar sus habilidades matemáticas básicas para el cálculo de dosis. Asimismo, Eastwood ${ }^{(14)}$, en estudiantes australianos de enfermería de segundo año, identificó deficiencias en la capacidad matemática de enfermeras calificadas y estudiantes: a) conceptuales, b) aritméticas y c) computacionales. De acuerdo al investigador, estas equivocaciones implican no poder formular una ecuación a partir de la información dada, no poder operar una ecuación dada, o equivocaciones aritméticas simples respectivamente. El estudio concluyó que algunos estudiantes de enfermería tienen deficiencias en la realización de cálculos precisos, con equivocaciones aritméticas y 
Fajardo Zapata AL, Caicedo Murte NP

conceptuales, lo que indica fallas fundamentales en su comprensión matemática y demuestran un nivel inaceptable para la práctica clínica de forma segura.

Se han buscado estrategias para minimizar o eliminar las equivocaciones en el cálculo de dosificación de medicamentos. Así, Karabağ ${ }^{(15)}$, en Turquía en un estudio que buscó evaluar nuevas herramientas para mejorar la habilidad de las estudiantes de enfermería en el cálculo de la dosis de medicamentos con instrucciones las basadas en la Web; encontró que los resultados de la enseñanza basada en el autoaprendizaje a través de Internet mejoraron las habilidades aritméticas y de cálculo de dosis de medicamentos. Hubo una correlación positiva entre la habilidad aritmética y el cálculo de la dosis del fármaco. De igual forma, Mackie et al. ${ }^{(6)}$, en Canadá, en un estudio cuyo objetivo fue determinar las áreas de desafío para los estudiantes en la realización de cálculos de dosis de medicamentos para diseñar intervenciones que mejoren esta habilidad, evaluó las fortalezas y debilidades en la enseñanza y el aprendizaje de los cálculos de dosis de medicamentos. Los datos se utilizaron para crear intervenciones en línea que luego se midieron para determinar el impacto en la capacidad del alumno para realizar los cálculos. El estudio concluyó que, con un esfuerzo concertado y un enfoque interdisciplinario para apoyar a los estudiantes de enfermería, se puede mejorar la habilidad para el cálculo de las dosis de medicamentos.

Por otra parte, Grugnetti ${ }^{(16)}$, en Italia, en una investigación realizada para evaluar la efectividad de un taller enfocado en mejorar las habilidades del cálculo de dosis de medicamentos en estudiantes de segundo año de enfermería, y cuyo fin era el de promover la seguridad en la administración de medicamentos, encontró que hubo una mejora significativa después de la intervención. El estudio concluyo que los talleres de habilidades clínicas pueden adaptarse para incluir técnicas de enseñanza que fomenten el desarrollo de habilidades de cálculo de dosis de medicamentos, y que estas estrategias de capacitación pueden mejorar la comprensión del cálculo de dosis de medicamentos en los estudiantes. Agregado a lo anterior, Wright $^{(17)}$ en Inglaterra, en un estudio que tuvo por objetivo evaluar las estrategias educativas para desarrollar y retener las habilidades en el cálculo de dosis de medicamentos por parte de las estudiantes de enfermería, con una metodología cuasiexperimental en dos grupos de estudiantes que recibieron diferentes enfoques para mejorar las habilidades en este campo, encontró que las estrategias implementadas hicieron la diferencia en la retención de las habilidades de cálculo de medicamentos en los estudiantes del grupo de intervenido. El estudio concluyó que parece haber evidencia que sugiere que las estrategias de enseñanza y aprendizaje correctas producen una mejor retención de las habilidades de cálculo de medicamentos. También en Australia, Ramjan ${ }^{(18)}$, identificó estrategias y predictores potenciales que ayudaran a las profesoras de enfermería a adaptar sus métodos de enseñanza y evaluación sobre el cálculo de medicamentos. Dicha exploración partió de las percepciones de los estudiantes sobre las intervenciones implementadas, que incluyeron pruebas de práctica en línea, escenarios de cálculo de medicamentos simulados de práctica clínica, 
Análisis de las habilidades matemáticas para el cálculo de dosis ...

pruebas escritas contextualizadas, remediación didáctica mejorada visualmente y talleres prácticos contextualizados, y encontró que los estudiantes de enfermería preferían un enfoque de aprendizaje práctico y contextualizado que fuera "auténtico" y alineado con la práctica clínica. Las intervenciones ayudaron a apoyar el aprendizaje de los estudiantes y a mejorar la confianza en el cálculo. El estudio concluyó que la implementación de la enseñanza y la evaluación de aritmética intensiva y contextualizada dentro de las instituciones universitarias mejoran el aprendizaje y promueven mejores prácticas de enseñanza.

Los estudios revisados han evidenciado deficiencias conceptuales y aritméticas en el cálculo de dosis de medicamentos en el personal de enfermería en formación. Dentro de las deficiencias algunos estudios han mostrado que los estudiantes de esta área de la salud tienen dificultades con los principios básicos de matemáticas básicas sobre todo evidencian una comprensión débil del sistema numérico decimal que lo que los lleva a cometer equivocaciones a la hora de realizar los cálculos pertinentes. También algunos estudios reflejaron una falta de congruencia entre el ámbito universitario y el clínico y una formación académica en matemáticas alejada de la realidad práctica. Lo anterior evidencia la necesidad de intervenciones en el aprendizaje para mejorar las habilidades matemáticas básicas en el cálculo de las dosis de medicamentos y la necesidad de cambiar las estrategias educativas en los currículos de enfermería para que se den en entornos realistas y simulados, además se deben reforzar de manera constante estas habilidades.

Por lo expresado anteriormente, es muy importante profundizar más en los aspectos propios de la habilidad de las matemáticas básicas en el quehacer de los futuros profesionales de la enfermería, que puedan explicar las equivocaciones en la dosificación de los medicamentos y en el cumplimiento de órdenes médicas, a fin de establecer los correctivos necesarios para reducirlos o eliminarlos. Si bien se han explorado las causas de algunos de ellos, es posible que existan otras que no han sido investigadas y que pudieran estar contribuyendo de manera importante en la problemática. Sin duda, investigaciones en esta dirección ayudaran a mejorar las estrategias de educación de los estudiantes de enfermería en cuanto a la seguridad en la administración y dosificación de los medicamentos.

El objetivo de la presente investigación fue evaluar las habilidades para el cálculo de las dosis de medicamentos en los estudiantes de enfermería de la fundación universitaria del Área Andina de Bogotá (Colombia).

¿Los estudiantes de enfermería de la Fundación Universitaria del Área Andina de Bogotá(Colombia) desarrollan las habilidades matemáticas para realizar los cálculos correctos de las dosis de medicamentos durante su proceso de formación?

\section{MATERIALES Y MÉTODOS}

Estudio descriptivo transversal orientado a evaluar las habilidades para el cálculo de las dosis de medicamentos en estudiantes de enfermería de la Fundación Univer- 
Fajardo Zapata AL, Caicedo Murte NP

sitaria del Área Andina, Institución de educación superior de carácter privado, ubicada en la ciudad de Bogotá (Colombia). Los estudiantes participantes en el estudio fueron jóvenes con edades comprendidas entre los 18 y 24 años, en su gran mayoría de sexo femenino. Se tomó como error el cinco por ciento (5\%); como se desconocía la prevalencia de las equivocaciones en las habilidades para las matemáticas básicas, se tomó como valor el $50 \%(\mathrm{p}=0,5)$. La población total de estudiantes fue de 1843 (considerados los 8 semestres académicos que componen el programa). La muestra se estimó en 256 estudiantes, corregida para población finita. Se dividió el tamaño total de muestra entre el número de semestres académicos para realizar una distribución equitativa entre los 8 semestres académicos, lo cual arrojó que se seleccionarían 32 estudiantes por semestre. Se tomó en consideración que el documento de identidad terminara en cifra par para hacer uniforme la selección del estudiante que participaría en el estudio. Se aplicó un instrumento denominado "Habilidad matemática para el cálculo de las dosis de medicamentos" que estuvo compuesto por 13 preguntas abiertas, que evaluaron los siguientes aspectos: manejo de la proporcionalidad, manejo de conversiones de unidades (manejo de fracciones decimales), manejo de porcentajes, manejo de volúmenes, comprensión de situaciones matemáticas que involucran la terminología, y la interpretación de conceptos matemáticos básicos mediante gráficos. Para evaluar la validez y confiabilidad del instrumento se usó el método de Kuder Richarson $\left(\mathrm{KR}_{20}\right)$, que es la prueba estadística determinada y validada que se aplica a cuestionarios de ítem dicotómicos y cuando existen alternativas con respuestas correctas e incorrectas y cuyo resultado fue de 0,82 lo cual indicó una buena consistencia interna. También el instrumento se sometió a la evaluación imparcial de tres profesores de matemáticas (juicio de expertos) quienes lo revisaron en cuanto a coherencia, claridad y pertinencia de cada una de las preguntas de acuerdo al objetivo de la investigación y se le realizó una prueba piloto con 10 estudiantes de diferentes semestres de enfermería de la misma institución, a quienes se les dio un tiempo de 30 minutos para resolver cada una de las situaciones planteadas en la prueba y se permitió utilizar calculadora (no se colocaron respuestas para que fueran marcadas por los estudiantes, sino que la prueba consistía en la resolución de la situación). Se realizaron los correctivos pertinentes y se adoptó el cuestionario. Se solicitó permiso a los profesores que estaban en clase con los respectivos grupos para la aplicación de la prueba. Las respuestas a cada una de las preguntas abiertas se codificaron como: si: resolvió correctamente la pregunta, no: no resolvió correctamente la pregunta. La codificación de las respuestas fue llevada a cabo de forma manual por el investigador. No se tomaron en consideración para el estudio las variables sociodemográficas, ni estudios previos de los participantes. Se aplicaron como criterios de inclusión: manifestar expresamente el deseo de participar voluntaria y autónomamente en el estudio, y ser estudiante de enfermería de cualquiera de los ocho semestres académicos de la Institución en la sede Bogotá y como criterios de exclusión: 
Análisis de las habilidades matemáticas para el cálculo de dosis ...

manifestar expresamente el deseo de no participar voluntaria y autónomamente en el estudio y no ser estudiante de enfermería de la Institución universitaria. No se realizó ninguna intervención durante el desarrollo del estudio. Se dio un tiempo de 30 minutos para la resolución de la prueba y se permitió usar la calculadora. La aplicación de la prueba para todos los estudiantes participantes se realizó en el lapso de una semana. La presente investigación se llevó a cabo respetando en todo momento las directrices de la declaración de Helsinki y en atención a la dignidad humana y a los derechos de las personas asimismo con el debido consentimiento de los participantes y con el conocimiento de las leyes colombianas que rigen los procedimientos de una investigación con participantes humanos. El estudio fue aprobado por el comité de ética de la Fundación Universitaria del Área Andina, los participantes firmaron previamente el consentimiento informado. Para la elaboración de la investigación se realizó un estudio de frecuencias y determinación de prevalencias mediante un análisis univariado. Si bien el presente estudio no es de tipo analítico y no buscó contrastar hipótesis, se planteó asociación con el ánimo de profundizar más en los hallazgos obtenidos mediante la prueba la prueba Chi cuadrado. El manejo de la información fue anónimo y confidencial. Los análisis estadísticos se llevaron a cabo en EpiInfo 7.2.0.1 y Minitab. La base de datos se elaboró en Excel.

\section{RESULTADOS}

La Tabla 1 muestra que, solo dos habilidades tuvieron desempeño por debajo del $50 \%$. el concepto matemático básico que mejor comprendieron e interpretaron los estudiantes de enfermería fue el manejo de la proporcionalidad. Se observó también que el manejo de las conversiones de unidades fue mejorando en la medida que avanzaban los semestres académicos, al igual que el manejo del concepto de los volúmenes. La comprensión de situaciones matemáticas básicas en dosificación de medicamentos fue muy deficiente en todos los semestres académicos considerados $(30,7 \%)$.

En cuanto a la interpretación de conceptos matemáticos mediante gráficos se advirtió que los estudiantes comprenden medianamente la información presentada en esta forma, ya que solamente el $50,2 \%$ de ellos respondió acertadamente las preguntas que evaluaron este ítem (Tabla 1).

Este estudio encontró asociación, entre el semestre académico y la habilidad en matemáticas básicas al efectuar el cálculo de las dosis de medicamentos donde se involucran el manejo de la proporcionalidad matemática $(\mathrm{p}=0,03)$. Asimismo, se observó asociación entre el semestre académico y el nivel de habilidad matemática en situaciones que implican el manejo de fracciones decimales básicas por parte de los estudiantes de enfermería $(\mathrm{p}=0,0002)$. Sumado a lo anterior, no se encontró diferencia entre ninguno de los semestres académicos en cuanto a la resolución de problemas de dosificación de medicamentos, ya que como se evidenció, los estudiantes de los ocho semestres académicos obtuvieron desempeños muy deficientes cuando se les solicitó que resolvieran situaciones clínicas, que implicaban el uso de las habilidades matemáticas básicas. 
Fajardo Zapata AL, Caicedo Murte NP

Tabla 1. Síntesis del porcentaje de respuestas acertadas emitidas por los estudiantes evaluados por semestre académico del programa de enfermería.

\begin{tabular}{|c|c|c|c|c|c|c|c|c|c|}
\hline & $\begin{array}{c}\text { Semes- } \\
\text { tre }\end{array}$ & $\begin{array}{c}\text { Semes- } \\
\text { tre }\end{array}$ & $\begin{array}{c}\text { Semes- } \\
\text { tre }\end{array}$ & $\begin{array}{c}\text { Semes- } \\
\text { tre }\end{array}$ & $\begin{array}{c}\text { Semes- } \\
\text { tre }\end{array}$ & $\begin{array}{c}\text { Semes- } \\
\text { tre }\end{array}$ & $\begin{array}{c}\text { Semes- } \\
\text { tre }\end{array}$ & $\begin{array}{c}\text { Semes- } \\
\text { tre }\end{array}$ & Total \\
\hline Ítem & 1 & 2 & 3 & 4 & 5 & 6 & 7 & 8 & $\%$ \\
\hline $\begin{array}{l}\text { Manejo de la } \\
\text { proporcionalidad }\end{array}$ & 90,6 & 75 & 75 & 87,5 & 81,1 & 90,6 & 82,8 & 87,5 & $85,4 \%$ \\
\hline $\begin{array}{l}\text { Manejo de } \\
\text { conversiones de } \\
\text { unidades (manejo } \\
\text { de fracciones } \\
\text { decimales) }\end{array}$ & 43,8 & 65,6 & 45,3 & 64,1 & 73,4 & 64,1 & 76,6 & 73,4 & $63,3 \%$ \\
\hline $\begin{array}{l}\text { Manejo de } \\
\text { porcentajes }\end{array}$ & 29,7 & 46,9 & 32,8 & 42,2 & 54,7 & 53,1 & 39,1 & 37,5 & $42 \%$ \\
\hline $\begin{array}{l}\text { Manejo de } \\
\text { volúmenes }\end{array}$ & 42,2 & 65,6 & 39 & 54,7 & 70,3 & 62,5 & 64,1 & 59,4 & $57,2 \%$ \\
\hline $\begin{array}{l}\text { Comprensión de } \\
\text { situaciones } \\
\text { matemáticas } \\
\text { básicas en } \\
\text { dosificación }\end{array}$ & 17,2 & 30,2 & 17,7 & 30,2 & 49 & 36,5 & 39,6 & 26 & $30,7 \%$ \\
\hline $\begin{array}{l}\text { Interpretación } \\
\text { conceptos } \\
\text { matemáticos } \\
\text { básicos mediante } \\
\text { gráficos. }\end{array}$ & 45,3 & 56,3 & 43,8 & 39,1 & 53,1 & 60,9 & 50 & 53,1 & $50,2 \%$ \\
\hline
\end{tabular}

Fuente: Elaboración propia utilizando los datos de las respuestas emitidas por los estudiantes de enfermería de la Fundación Universitaria del área Andina.

\section{DISCUSIÓN}

Si bien hay que resaltar que en este estudio encontró que solo dos habilidades tuvieron desempeño por debajo del 50\%, los estudiantes de pregrado de enfermería de la Fundación universitaria del área Andina que participaron en el estudio tuvieron dificultades para entender situaciones matemáticas en donde se involucraban conceptos básicos de matemáticas. Pero tal vez, lo que más llamó la atención fueron las serias deficiencias en la resolución de situaciones matemáticas en la dosificación de medicamentos, donde solo el 30,7\% de los estudiantes logró resolver las situaciones que se les plantearon; también el manejo de los porcentajes mostró difi- cultades, ya que únicamente el 42\% logró resolver la situación planteada. En la interpretación conceptos matemáticos básicos mediante gráficos, apenas el 50,2\% logró interpretar la situación propuesta.

Los resultados arrojados por este estudio coinciden con los obtenidos por Bagnasco $^{(13)}$ en Estados Unidos, quien en su estudio encontró que, los estudiantes universitarios de enfermería tenían dificultades principalmente con los principios básicos de matemáticas; Así mismo Yapucu $^{(12)}$, en Turquía, que en una investigación que buscaba conocer las habilidades para el cálculo de medicamentos en estudiantes de dos escuelas de enfermería, 
Análisis de las habilidades matemáticas para el cálculo de dosis ...

encontró que estos tenían bajas habilidades para el cálculo de medicamentos, lo cual también coincide con los hallazgos de esta investigación. También Grugnetti ${ }^{(12)}$, en Italia, en un estudio para verificar si el uso de la calculadora en una prueba de habilidad matemática escrita reducía las equivocaciones en el cálculo de dosis de medicamentos en estudiantes de enfermería, reportó serias deficiencias en los cálculos matemáticos a pesar del uso del dispositivo, lo cual coincide con los hallazgos de este estudio que también permitió el uso de la calculadora para resolver las situaciones matemáticas planteadas. Así mismo Grandell ${ }^{(19)}$, en Finlandia, encontró deficiencias en las habilidades en matemáticas básicas que no les permitieron a las estudiantes de enfermería realizar cálculos de dosis de medicamentos con exactitud, lo cual coincide con lo hallado en la presente investigación.

Al parecer las deficiencias en el cálculo de dosis de medicamentos no son solamente un problema de las estudiantes de enfermería de la Fundación Universitaria del Área Andina donde se realizó el estudio, ya que Wirtz (20) en un estudio observacional de las equivocaciones en la medicación intravenosa en el Reino Unido y en Alemania, en estudiantes y enfermeras recién graduadas, encontró que el porcentaje de equivocaciones fue estadísticamente significativo. Si bien el presente estudio no se realizó en el ámbito clínico, los estudiantes de séptimo y octavo semestre del programa de enfermería mostraron un desempeño deficiente, lo cual debe preocupar porque éstos están a las puertas de ingresar al mundo laboral. También los hallazgos de esta investígación coinciden con los de Eastwood et al. ${ }^{(14)}$, en Australia, quien en un estudio cuyo objetivo fue determinar si una muestra de estudiantes australianos de enfermería de segundo año, podría calcular con precisión las dosis de medicamentos y realizar algunos cálculos matemáticos básicos, encontró que, algunos tenían deficiencias en la realización de cálculos precisos, con equivocaciones aritméticas y conceptuales, lo que de acuerdo al autor, indica fallas fundamentales en su comprensión matemática y demuestran un nivel inaceptable para la práctica clínica de forma segura. Estos hallazgos también son coincidentes con los de Fleming ${ }^{(21)}$, quien encontró que el tipo más frecuente de equivocación cometido por los profesionales de enfermería en el cálculo de las dosis, correspondían a equivocaciones conceptuales de su proceso educativo. Por otra parte, Rainboth et al. ${ }^{(22)}$ en un estudio para determinar las habilidades de cálculo matemático de estudiantes de enfermería, concluyeron que las equivocaciones de medicación son comunes y que uno de los problemas residía en que los sistemas de administración de medicamentos eran defectuosos, lo cual reafirma los hallazgos de la investigación realizada. Más recientemente, Özyazıcıoğlu ${ }^{(10)}$, en Turquía en un estudio cuyo objetivo fue evaluar el conocimiento de estudiantes de enfermería sobre los cálculos de dosis pediátricas, encontró que los estudiantes contestaron correctamente el $87,1 \%$ de las preguntas, mientras que el $9,5 \%$ respondió incorrectamente y el 3,4\% dejó el test en blanco. Se encontró que los estudiantes fallaron en el cálculo cuando había que utilizar fracciones decimales. Si bien los resultados difieren en porcentaje a los 
Fajardo Zapata AL, Caicedo Murte NP

encontrados por la presente investigación, también reafirman que uno de los puntos débiles en los conocimientos de las matemáticas básicas son las fracciones decimales, factor que fue uno de los hallazgos de la presente investigación.

Finalmente se puede afirmar que la seguridad del paciente en el ámbito clínico debe ser una prioridad y que el cálculo de la dosis exacta de los medicamentos a administrar por parte de estudiantes $y$ enfermeras graduadas debe ser una constante preocupación de las instituciones educativas y de las instituciones de salud, para que no se cometan equivocaciones que puedan poner en riesgo la salud y vida de los pacientes. Los estudios revisados han evidenciado deficiencias conceptuales en matemáticas básicas para el cálculo de dosis de medicamentos en estudiantes de enfermería, no solamente de la Institución de educación superior donde se realizó el estudio, sino de varios Instituciones educativas de países del mundo en los que se han realizado estudios similares.

Lo anterior evidencia la necesidad de intervenciones en el aprendizaje para mejorar las habilidades matemáticas básicas en el cálculo de las dosis de medicamentos y la necesidad de cambiar las estrategias educativas en los currículos de enfermería para que se desarrollen en entornos realistas y simulados, y que, además, se deben reforzar de manera constante estas habilidades.

Por otra parte, Sherriff et al. ${ }^{(23)}$, argumentan que no hay suficiente evidencia para sugerir que las deficiencias en la competencia para las matemáticas de los profesionales de enfermería sean causantes de equivocaciones en la medicación, y que se requieren más investigaciones para determinar la solidez de los procesos actuales para evaluar la competencia de cálculo de medicamentos de estas profesionales. Lo anterior está en contraposición con los hallazgos de la presente investigación y con la consulta bibliográfica realizada en diferentes bases de datos donde los estudios reportaron serias deficiencias en el cálculo de dosis de medicamentos no solamente de estudiantes de enfermería, sino también de enfermeras ya graduadas. Asimismo, Savaje ${ }^{(24)}$, en Tanzania concluyó en su estudio que se requiere educación continuada y planificada del personal de enfermería para mejorar la habilidad en la dosificación de medicamentos, conclusión que es coherente con las conclusiones emitidas por el presente estudio.

Es de anotar que el estudio presentó algunas limitaciones en cuanto al posible sesgo en la elección de la muestra población estudiantil, ya que solo tomó en consideración una sola institución educativa, además de ser privada. Es posible que, si se realiza el estudio con Poblaciones que involucren estudiantes de otras universidades privadas y se contemple la participación de la universidad pública, así como un número mayor de estudiantes, los resultados pudieran ser diferentes a los encontrados en el presente estudio. También se puede contemplar alguna limitación en el diseño del instrumento de investigación, que, si bien trato de abarcar temas sensibles en las habilidades matemáticas que deben poseer los estudiantes de enfermería, es probable que no haya alcanzado a abarcar toda dimensión que se hubiera querido para realizar esta investigación. 
Análisis de las habilidades matemáticas para el cálculo de dosis ...

\section{CONCLUSIONES}

Esta investigación dejo conocer algunos aspectos representativos de las habilidades en matemáticas básicas por parte de los estudiantes universitarios del programa de enfermería de la Fundación universitaria del Área Andina y permitió develar falencias en el cálculo de dosificación de los medicamentos, aspecto fundamental en el ámbito clínico en el cual desarrollarán sus actividades en un futuro los actuales estudiantes de esta área de la salud. Al profundizar en el análisis de los resultados arrojados por la aplicación del instrumento de investigación, sin lugar a dudas surge la imperiosa necesidad de mejorar los procesos en matemáticas que permitan un adecuado desempeño por parte de los futuros profesionales, y a su vez revisar los actuales procesos educativos en el área de matemáticas básicas, que implican no solamente al estudiantado sino también a la formación de los profesores en educación superior.

Adicionalmente es muy importante que los profesores propicien estrategias que permitan que el estudiante integre a su conocimiento todas las habilidades de las matemáticas básicas para que así pueda dar cumplimiento a los lineamientos que tienen que ver con el aprendizaje, razonamiento, resolución y planteamiento de problemas, comunicación, modelación, colaboración, comparación y ejercitación de los procesos y además establezcan el desarrollo del pensamiento matemático con los sistemas propios de las matemáticas.

Además, resulta primordial que los profesores del área de matemáticas comprendan que el aprendizaje de esta ciencia está estrechamente entrelazado con las actividades clínicas propias del quehacer del profesional de enfermería, por lo tanto, es importante el uso de métodos de enseñanza innovadores $\mathrm{y}$ efectivos para mejorar el rendimiento de los estudiantes y su compromiso con las matemáticas a nivel universitario, particularmente en el primer año. El desarrollo de las habilidades matemáticas y su relación con la vida cotidiana y con el desarrollo de las actividades laborales sin duda, ayudará a los estudiantes a apreciar la importancia de esta disciplina.

Hay que adicionar que, las dificultades en la dosificación de medicamentos están relacionadas con una formación educativa restringida, con expectativas poco realistas sobre lo que implica el estudio de la enfermería, las demandas de la educación superior, los ambientes de aprendizaje y de enseñanza universitaria $\mathrm{y}$, en nuestro medio con la precaria situación económica, que obliga al estudiante a trabajar y estudiar lo cual incide en un bajo rendimiento académico. Además, se deben considerar los resultados obtenidos en la investigación a la luz de una posible deficiencia en la formación matemática en la educación primaria y secundaria, es decir, el problema encontrado por la investigación, puede tener profundas raíces estructurales de nuestro sistema educativo que se hacen evidentes en la educación universitaria.

\section{AGRADECIMIENTOS}

El autor quiere expresar sus agradecimientos al Dr. José Luis Ángel Rodríguez Silva, por sus valiosos aportes a este trabajo de investigación.

Este proyecto fue parcialmente financiado por la Fundación universitaria 
Fajardo Zapata AL, Caicedo Murte NP

del Área Andina. El resto de los costos fueron asumidos por los autores.

\section{REFERENCIAS BIBLIOGRÁFICAS}

${ }^{1}$. Organización Mundial de la Salud OMS. [Internet]. Ginebra, Suiza: 10 datos sobre la seguridad del paciente; 2018 [citado el 17 de octubre de 2020]. Disponible en

https://www.who.int/es/news$\mathrm{room} /$ fact-sheets/detail/patient-safety

2. Alba A. Errores de enfermería en la atención hospitalaria. Revista Enfermería Instituto Mexicano de Seguro Social. 2011;19(3): 149-154.

3. Ewig C. Occurrence of Potential Adverse Drug Events from Prescribing Errors in a Pediatric Intensive and High Dependency Unit in Hong Kong: An Observational Study. Pediatr Drugs. 2017; 19(4): 347-355.

4. Di Muzio MD, Di Simone E, y Cianfrocca C. Can nurses' shift work jeopardize the patient safety? A systematic review. Can nurseEur Rev Med Pharmacol Sci. 2019; 23 (10): 4507-4519.

5. Gorgich E. B. Investigating the Causes of Medication Errors and Strategies to Prevention of Them from Nurses and Nursing Student Viewpoint. Glob J Health Sci. 2016; 8(8): 544-48.

6. Mackie J, y Bruce CD. Increasing nursing students' understanding and accuracy with Medical dose calculations: A collaborative approach. Nurse Educ Today. 2016; 40: 146-53.

7. Franco Da Silva E, De Faveri F, y Lorenzini L. Errores de medicación en el ejercicio de la enfermería: una revisión integrativa. Enfermería global.2014; 13(34).
${ }^{8 .}$ Institute of Medicine (US) Committee on Quality of Health Care in America. To Err is Human: Building a Safer Health System. Kohn LT, Corrigan JM, Donaldson MS, editors. Washington (DC): National Academies Press (US); 2000. [citado el 12 de noviembre de 2020]. Disponible en https://pubmed.ncbi.nlm.nih.gov/2507 $7248 /$

9. Coyne E. Enhancing student nurses' medication calculation knowledge; integrating theoretical knowledge into practice. Nurse Educ Today. 2013; 33(9): 1014-1019.

10. Özyazıcıoğlu N, Aydın Aİ, Sürenler S, Çinar HG, Yılmaz D, Arkan B, Tunç GÇ. Evaluation of students' knowledge about paediatric dosage calculations. Nurse Educ Pract. 2018; 28: 34-39.

${ }^{11 .}$ McMullan M. Patient safety: numerical skills and drug calculation abilities of nursing students and registered nurses. Journal of Advanced Nursing. 2010; 66: 891-899.

12. Yapucu U. Mathematical and Drug Calculation Skills of Nursing Students in Turkey. International Journal of Caring Sciences. 2016; 9(1): 200-227.

13. Bagnasco A. Mathematical calculation skills required for drug administration in undergraduate nursing students to ensure patient safety: A descriptive study: Drug calculation skills in nursing students. Nurse education in practice. 2016; 16 (1): 33-39.

14. Eastwood KJ, Boyle MJ, Williams B, y Fairhall R. Numeracy skills of nursing students. Nurse Educ Today. 2011; 31(8): 815-8.

15. Karabağ A, y Dinc L. Effects of WebBased Instruction on Nursing Students' 
Análisis de las habilidades matemáticas para el cálculo de dosis ...

Arithmetical and Drug Dosage Calculation Skills. Comput Inform Nurs. 2017; 35(5): 262-269.

16. Grugnetti AB. Effectiveness of a Clinical Skills Workshop for drugdosage calculation in a nursing program. Nurse Educ Today. 2014; 34(4): 619-24.

17. Wright K. ¿Can effective teaching and learning strategies help student nurses to retain drug calculation skills? Nurse Educ Today. 2008; 28(7): 856-64.

18. Ramjan LS. Identifying strategies to assist final semester nursing students to develop numeracy skills: a mixed methods study. Nurse Educ Today. 2014; 34(3): 405-12.

19. Grandell, H. Medication Calculation Skills of Graduating Nursing Students in Finland. Advances Health Sciences Education. 2001; 6(1):15-24.

20. Wirtz V. An observational study of intravenous medication errors in the
United Kingdom and in Germany. Pharmacy World and Science. 2003; 25: 104-111.

21. Fleming S. An evaluation of de drug calculation skills of registered nurses. Nurse Education in Practice. 2014; 14:55-61.

22. Rainboth L, y De Masi C. Nursing students' mathematic calculation skills. Nurse Education in Practice. 2006; 6:347-353.

23. Sherriff K, Wallis M, y Burston S. Medication calculation competencies for registered nurses: a literature review. Australian Journal of Advanced Nursing. 2011; 28:75-83

24. Savaje AR. Educational audit on drug dose calculation learning in a Tanzanian school of nursing. Afr Health Sci. 2015; 15 (2): 647-55. 